\title{
Bestattungspraxis: Figurationen im Wandel
}

Bestattungsrituale dienen dazu, Verstorbene von den Lebenden abzutrennen. Um uns als Lebende zu schützen, müssen wir sichergehen, dass die Toten fortbleiben. So geleiten wir sie ,hinüber', an eine imaginäre Schwelle, wir sprechen von ihrer Reise, Transformation oder Auflösung, wir schicken sie an einen Ort, den wir als Jenseits oder auch als Nichts bezeichnen - auf jeden Fall ein Ort, der uns Lebenden nicht zugänglich ist.

Rituale, mit denen man die Toten an jene Schwelle eskortiert, dienen auch der Vergewisserung ihres Todes: Gäben wir ihnen kein Geleit, so wären wir nicht sicher, ob sie wirklich physisch fort sind. Tote rituell zu erinnern - etwa indem man eine Kerze zum Gedenken anzündet oder eine Schachtel mit alten Fotos öffnet oder an Allerheiligen an einem Gottesdienst teilnimmt - trägt dazu bei, die Erinnerung an sie ins Leben zu integrieren. Gerade die Wiederholung der Rituale befördert auch den prozessualen Charakter, d. h. die dauerhafte Transformation solcher Erinnerung.

In den christlich geprägten westlichen Ländern ist die Bestattungspraxis aktuell in starkem Wandel begriffen, der sich in der Gestaltung von Abdankungsfeiern sowie im Umbau von analogen und in der permanenten Veränderung von digitalen Friedhöfen manifestiert.

Der Wandel liegt maßgeblich in der seit Jahrzehnten voranschreitenden allgemeingesellschaftlichen Abkehr von der Kirche begründet, gegenwärtig wird die kirchliche Hoheit über Bestattungsprozesse stark zurückgedrängt. Durch den Verzicht auf manche sakrale Ritualbestandteile ergeben sich Leerstellen, die neu ausgestaltet werden müssen. Dabei ist insbesondere eine Öffnung für neue Akteur:innen festzustellen: Die traditionelle Kernfamilie und die kirchlichen Vertreter:innen sind oft nicht mehr die einzigen, die bei heutigen Bestattungen einen Part übernehmen, denn die neue Ausgestaltung von Abschiedsritualen hat sich für weitere Mitwirkende geöffnet: Freund:innen, Arbeitskolleg:innen oder auch ehemalige Lebenspartner:innen nehmen heute ebenfalls eine aktiv gestaltende Rolle ein. Aber auch die digitalen Medien tragen zu Veränderungen in der Bestattungskultur bei, indem sie neue Gedenkkulturen und Trauerpraktiken ermöglichen und entsprechende kommerzielle Angebote befördern. 


\section{Ritual im Übergang: Wem gehören die Toten?}

Im Laufe des 19. und 20. Jahrhunderts hat die Kirche die Sterbebegleitung und Totenversorgung Schritt für Schritt an säkulare medizinische Einrichtungen abgetreten. Seit ein, zwei Jahrzehnten verliert sie nun zunehmend auch ihre führende Rolle bei Bestattungen. Heute besteht eine starke Tendenz zu Mischformen aus delegierten und nicht-delegierten Teilen einer Bestattung, bei denen die Kirche oftmals zwar durchaus noch involviert, aber nicht mehr allein für den inhaltlichen und dramaturgischen Ablauf zuständig ist. Die frühere, traditionell delegierte Bestattung, bei der ein Pfarrer vollumfänglich die Verantwortung übernahm, sollte Trauernde von Organisationsarbeit entlasten und ihnen feste Ritualformen anbieten, die sie in ihrer akuten Trauerkrise unterstützten. Die heute vorherrschende Tendenz zu Mischformen hingegen führt zu Patchwork-Ritualen, bei denen man auf gewisse Bestandteile der konventionellen kirchlichen Bestattung zurückgreift und sich aber zugleich die Möglichkeit für einen eigenen Aktionsraum verschafft.

Ein wesentliches Moment stellt dabei die Hoheit über das Wort dar. Anstelle von oder in Ergänzung zu Pfarrer:innen und Mitgliedern der Kernfamilie treten weitere Angehörige und Bekannte oder aber auch freie Bestattungsredner:innen oder Ritualbegleiter:innen vor das Trauerpublikum, um die verstorbene Person zu würdigen. Überdies übernehmen Hinterbliebene oftmals zumindest partiell die Regie der gesamten Bestattung, indem sie einzelne Bestandteile des Ablaufs festlegen und dabei etwa Redebeiträge und Musikstücke an Personen aus dem privaten Umfeld der Verstorbenen delegieren. Zunehmend werden die Zeremonien auch in nicht-kirchliche Räumlichkeiten verlagert. Durch diese Wandlungen treten individuelle Merkmale der verstorbenen Person, vor allen Dingen ihre persönlichen Beziehungen und selbstgewählten Zugehörigkeiten, deutlicher in den Vordergrund als bei standardisierten kirchlichen Abdankungen. Allerdings wirft dies eine Reihe neuartiger Fragen auf, die als Symptome der kirchlichen Ablösung gelesen werden können:

\section{Das Vorrecht von Deutungshoheit}

Redner:innen aus dem persönlichen Umfeld setzen die verstorbene Person unweigerlich so in Szene, wie sie sie gekannt haben. Dabei sind sie unter Druck darzulegen, dass sie eine besondere, eine gute und nahe Beziehung zur toten Person hatten und folglich wahrhaft über sie sprechen können. $\mathrm{Zu}$ diesem Zweck schildern sie gemeinsame Erlebnisse oder gemeinsam besuchte Orte, sie erzählen von ihrem Austausch mit der verstorbenen Person und lassen die Trauergemeinde an ihren inneren Bildern teilhaben. Indem sie 
derart aus ihrer je eigenen Perspektive möglichst Genaues über die Toten auszusagen versuchen, machen sie sich diese zu eigen. Mitglieder der Familie sind weniger unter Druck, ihre Nähe und intime Kenntnis der verstorbenen Person zu beweisen. Sie müssen sich die Verstorbenen nicht aneignen, da sie ihnen als Familienmitglieder zwangsläufig angehören. In allen Fällen bieten die Reden letzte veröffentlichte (Sprach-)Bilder der toten Person, welche auch entferntere Bekannte von der Trauerfeier mit nach Hause nehmen, die möglicherweise gar nichts von den erzählten bilateralen Beziehungen wussten. Wem also gehören die Toten, wer darf wie über sie sprechen, und wer nicht? Heute erhalten zumeist nahe $\mathrm{Zu}$ - und Angehörige der Verstorbenen das Vorrecht zu sprechen bzw. das Vorrecht auf Deutungshoheit. Im Unterschied zu einem Pfarrer oder einer Pfarrerin sprechen sie dabei oft mit brechenden Stimmen, mit unterdrückten oder laufenden Tränen, mit quälenden Pausen im Sprechen, in denen sie um Fassung ringen.

\section{Risse in der Trauercommunity}

Bei einer Trauerzeremonie kommen sehr unterschiedliche Leute zusammen, die eines gemeinsam haben, was sie für einen Moment zur Community macht: Sie alle haben die verstorbene Person gekannt, sie alle haben innere Bilder von ihr. Im Rahmen der neuen, nicht mehr gänzlich unter kirchlicher Hoheit stehenden Zeremonien treten zu diesen bestehenden inneren Bildern weitere, öffentlich und nicht selten recht ausgreifend verlautbarte Bilder von anderen Personen hinzu. Eine traditionelle kirchliche Bestattung enthält stets einen ruhig verlesenen genormten Lebenslauf der verstorbenen Person, zu dem man sich still und leise seine eigenen Gedanken machen kann; sie zeichnet sich dadurch aus, dass sich alle Trauernden anhand ihrer eigenen Bilder leise und einzeln, aber aufgehoben in der Trauercommunity, von der toten Person verabschieden können. Diese neuen Formen von Abschiedszeremonien aber erzeugen neuartige Risse in der Trauercommunity: Es entstehen Unterschiede und Asymmetrien, indem einzelne Personen als Redner:innen hervorgehoben werden, deren Andenken als wesentlicher, wahrer, mitteilenswerter und letztgültiger erscheint als dasjenige von anderen Mitgliedern der Trauercommunity.

\section{Keine Überantwortung der toten Person}

Ein zentraler Bestandteil der kirchlichen Bestattung besteht in der gemeinsamen Überantwortung der toten Person an Gott. Dies bleibt heute in vielen Fällen eine Leerstelle, d. h. es ereignet sich keine explizite Überantwortung mehr. An wen will man die Toten adressieren, wenn man an keinen gemeinsam geteilten Gott mehr glaubt? Wir wissen nicht, wohin mit den Toten. 
Diese Symptome basieren auf einer verlorenen christlich-religiösen Stabilität. Sie kennzeichnen die ,Bestattung im Übergang: eine unsichere, diffuse, nicht stabile, experimentelle Bestattungspraxis, die sich zunehmend verweltlicht, aber nach wie vor auch sakrale Elemente aufweist. Unsicher ist die Praxis deshalb, weil sie die Möglichkeit bestehen lässt, dass man es auch anders hätte machen können. Entsprechende Zweifel, ob man es richtig gemacht hat, gilt es heute auszuhalten. So wie man die Bestattung oder Teile davon nicht delegiert, so lassen sich auch diese Zweifel nicht delegieren. Das ,Ritual im Übergang، ist geprägt von Suchbewegungen und Experimenten, es vollzieht sich seit Jahrzehnten leise und schleichend, aber ohne wegweisendbegleitende öffentliche Debatten.

\section{Friedhöfe: analog, digital}

\section{Naturbestattungen und thematische Friedhofscluster}

Wie in der Ritualgestaltung bei Trauerzeremonien sind auch in der Gestaltung der Friedhöfe seit etwa zwei Jahrzehnten starke Veränderungen zu beobachten, in denen sich eine Abkehr von der kirchlichen Bestattungspraxis manifestiert. An erster Stelle ist der anhaltende Trend zu Naturbestattungen zu nennen, d. h. die Beisetzung von Urnen außerhalb von Friedhöfen. In der Schweiz wird dies durch eine liberale Gesetzgebung begünstigt, welche im Gegensatz zu umliegenden Ländern die Aschenbeisetzung auf Privatgrund oder in der freien Natur grundsätzlich erlaubt. Infolgedessen treten in der Schweiz regelmäßig neue Dienstleistungsangebote auf den Plan, die immer neue Touren für Ascheverstreuung in Bergen oder Seen vermitteln.

Eine beliebte Variante dieses Trends bieten die FriedWälder, für die frei zugängliche Teile eines natürlichen Waldes als letzte Ruhestätte kultiviert werden, wie beispielsweise der Friedwald Oberramsern in Solothurn (vgl. Abb. 78 ).

In einem solchen Waldareal kann man sich einen Baum aussuchen und kaufen, um sich dann später unter ihm beisetzen zu lassen. Oder man erwirbt einen Baum anlässlich eines Todesfalls, um ihn augenblicklich als Bestattungsort zu beanspruchen. Voraussetzung für eine Bestattung ist die Kremation; die Asche wird an den Baumwurzeln in die Erde eingelassen, wodurch man sich vorstellen mag, dass die verstorbene Person in dem Baum gleichsam sinnbildlich fortlebt. 


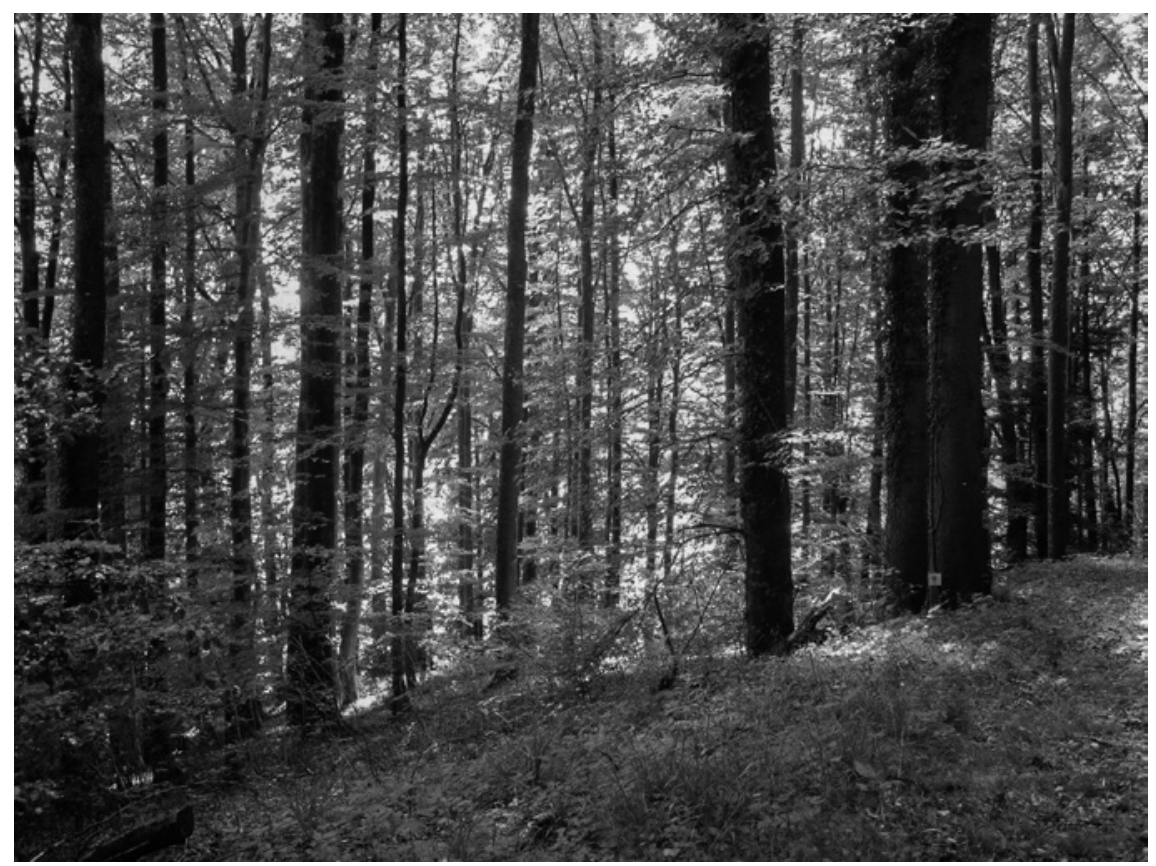

Abb. 78 FriedWald, Oberramsern-Messen, Solothurn. Online unter: https://www. solothurnerzeitung.ch/solothurn/kanton-solothurn/friedwald-statt-friedho f-immer-oefters-wird-ein-baum-einem-grabstein-vorgezogen-131548756\# (letzter Zugriff: 23.11.2021).

Das Nutzungsrecht an einem solchen Baum ist auf 99Jahre angelegt, womit die allgemeine Ruhefrist von 25 Jahren für ein Grab um ein Vielfaches überschritten wird.

FriedWälder erleben seit zwei Jahrzehnten einen anhaltenden Boom. Das Konzept des FriedWaldes wurde vom Schweizer Elektroingenieur Ueli Sauter patentiert, der Name ist markenrechtlich geschützt. In Deutschland und in der Schweiz gibt es mittlerweile Dutzende FriedWälder. ${ }^{1}$ Auch traditionelle Friedhöfe haben das Konzept kopiert, indem sie bestimmte Areale entsprechend umgewidmet haben. Beispiele hierfür sind der „Wald für Aschenbeisetzungen“ des Zürcher Friedhofs Hönggerberg oder die Baumgräber auf dem Hamburger Friedhof Ohlsdorf.

12012 gab es in der Schweiz 65 und in Deutschland 42 FriedWälder; 2021 waren es 8o $(\mathrm{CH})$ bzw. 76 (D). 
Ein weiterer Trend neben den Naturbestattungen sind verschiedene Formen von Individualisierungen innerhalb der kirchlichen Friedhöfe. So bieten diese spezielle Areale an, die bestimmten Gemeinschaften vorbehalten sind, etwa den Fans bestimmter Fußballvereine wie dem FC Schalke o4 oder dem HSV (vgl. Abb. 79 und 8o),

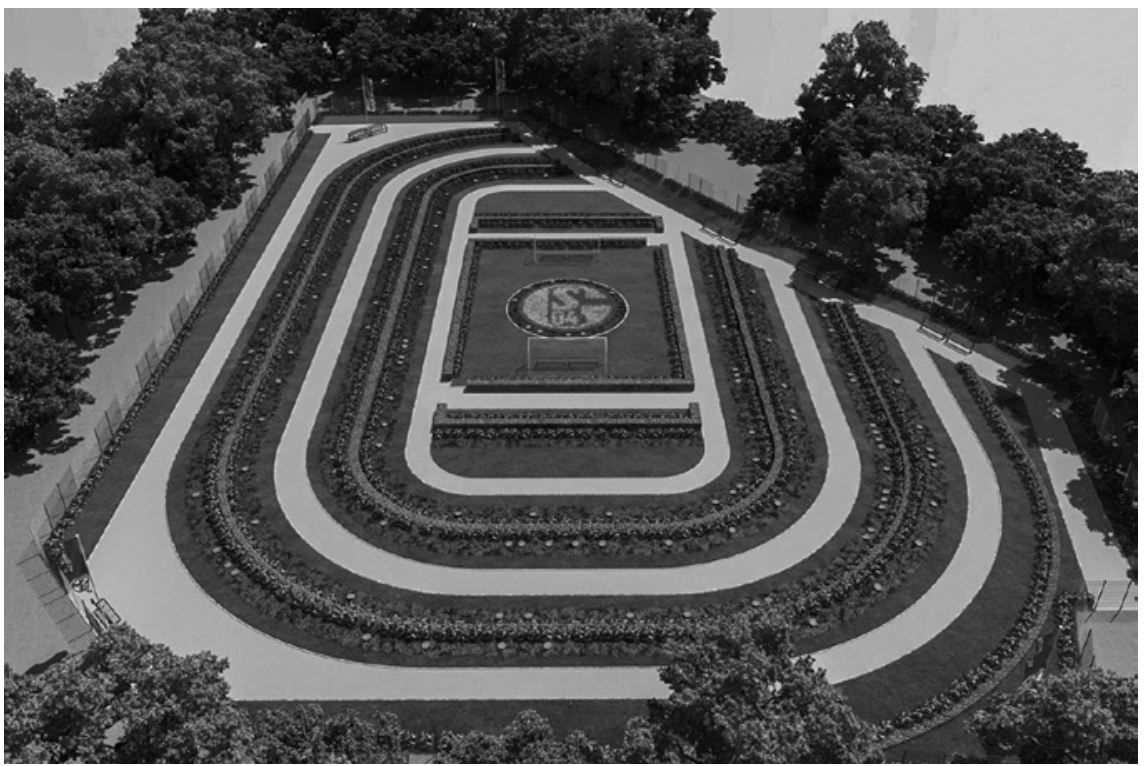

Abb. 79 Friedhof für FC Schalke o4-Fans, Gelsenkirchen, Friedhof Beckenhausen-Sutum. Online unter: https://www.welt.de/regionales/duesseldorf/galleryı1186o11o/SchalkeFans-haben-ihren-eigenen-Friedhof.html (letzter Zugriff: 23.11.2021)

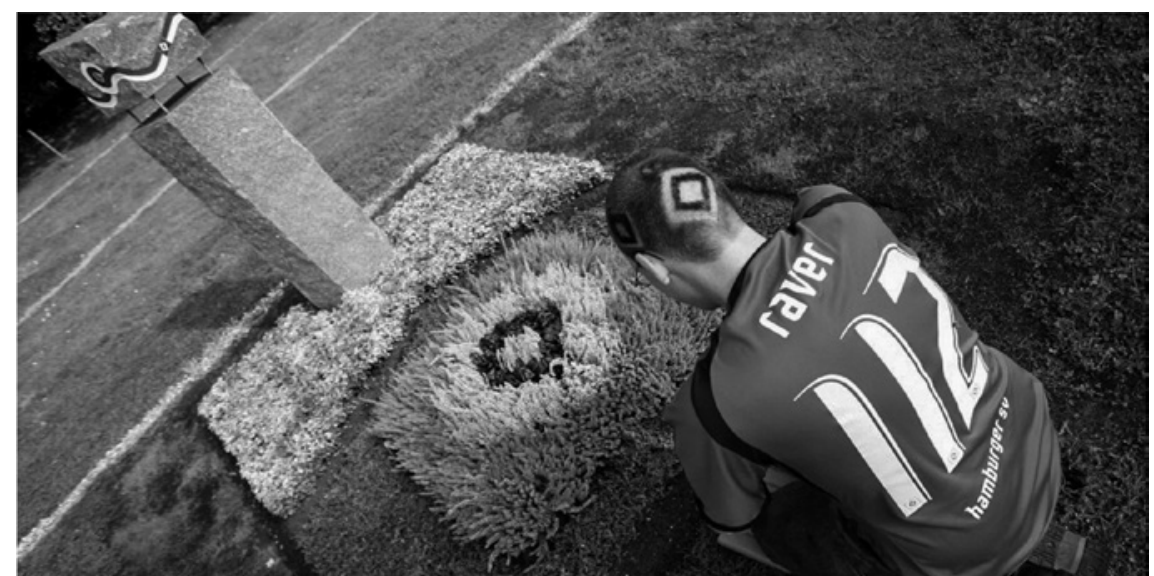

Abb. 8o HSV-Grabfeld, Friedhof Altona. Online unter: https://taz.de/Fan-Bestattunge n-sterben-aus/!54336o3/ (letzter Zugriff: 23.11.2021). 
oder sogenannten Sternenkindern (vgl. Abb. 81 und 82)

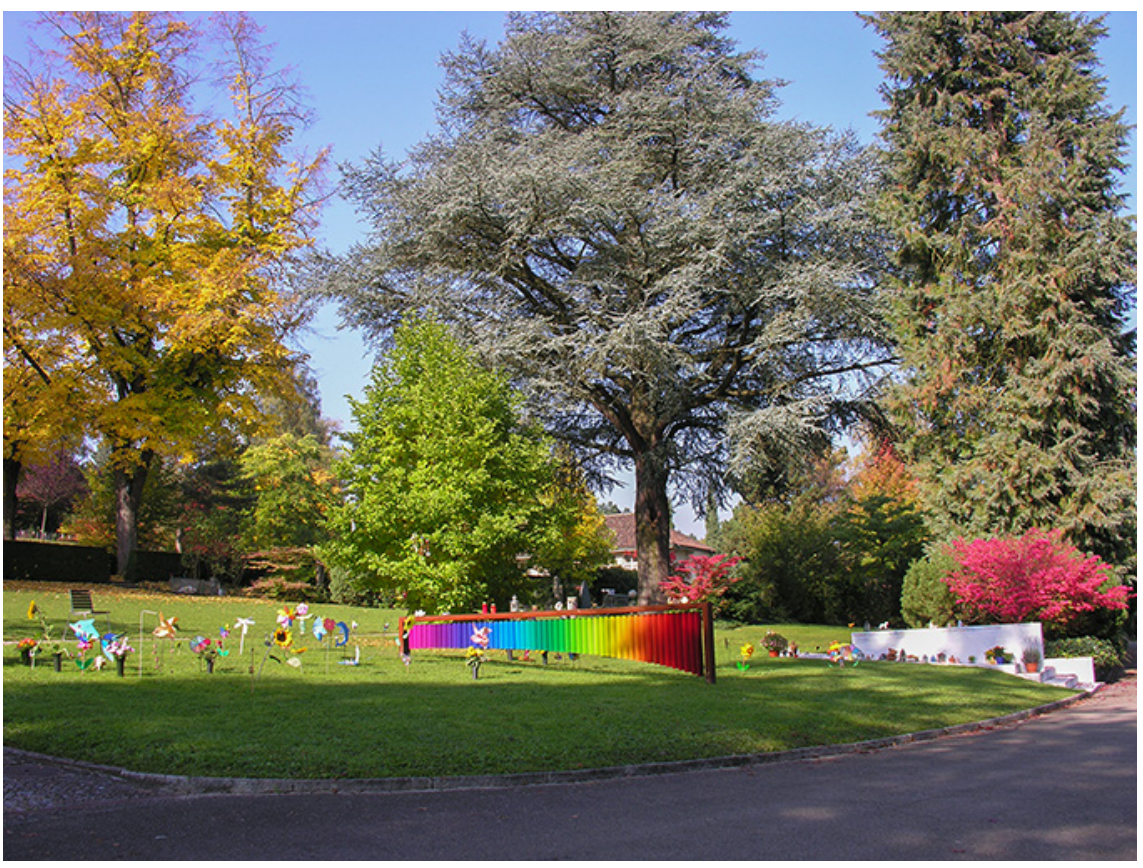

Abb. 81 Sternenkinder-Grabfeld, Friedhof Nordheim Zürich. ㄷ Bestattungsamt Zürich, 2021.

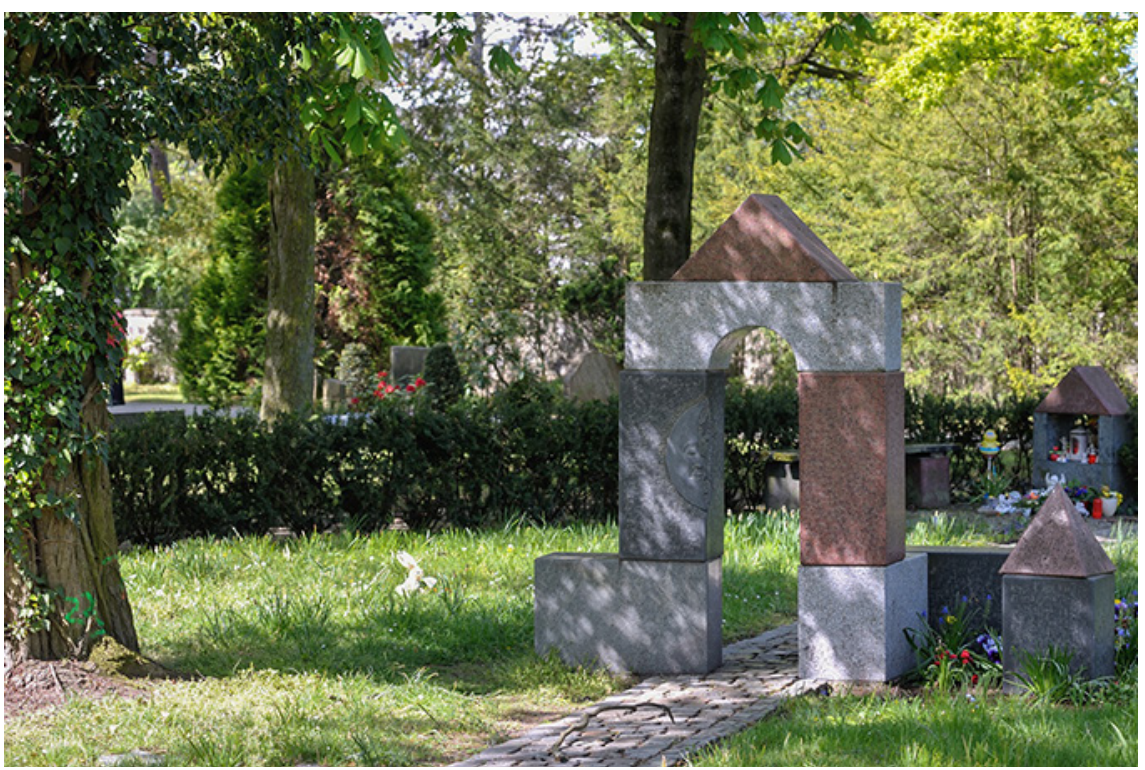

Abb. 82 Sternenkinder-Grabfeld, Friedhof Hanau. (C) Friedhof Hanau, 2021. 
oder auch Personen, die gemeinsam mit ihren Haustieren bestattet werden möchten (sogenannte Mensch-Tier-Bestattung) (vgl. Abb. 83).

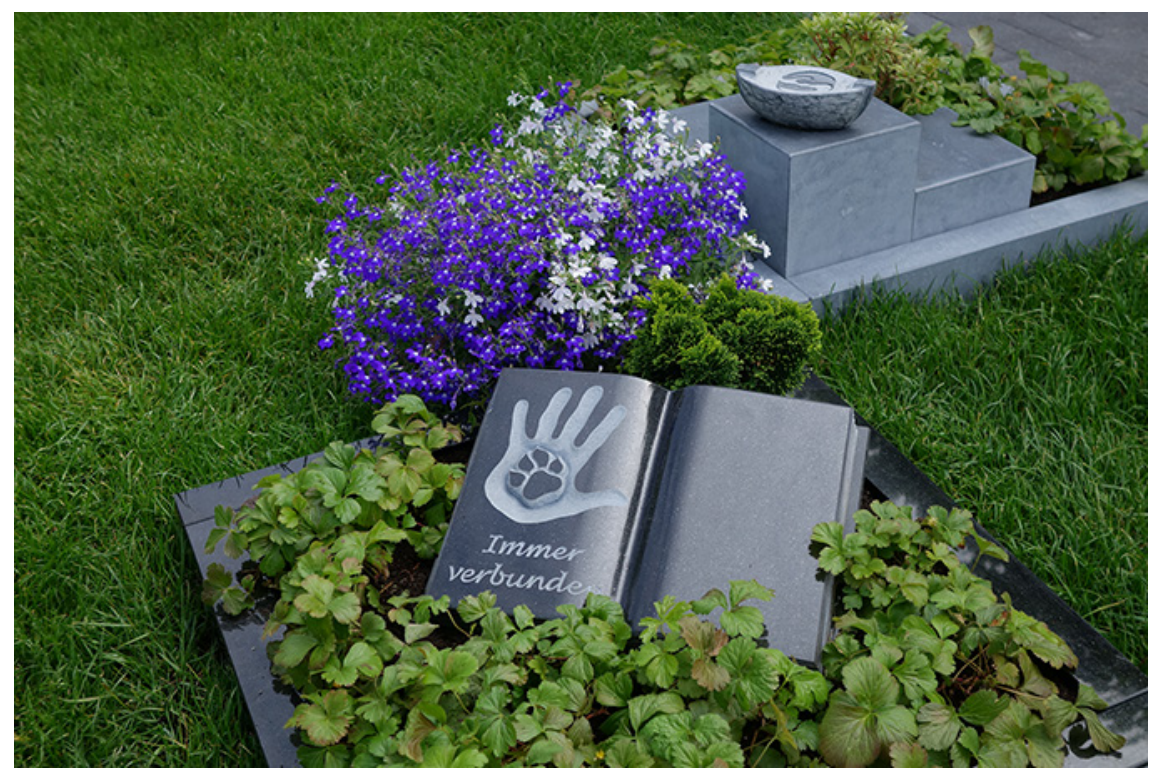

Abb. 83 Mensch-Tier-Bestattung, Friedhof Unser Hafen, Essen-Frintrop. (C) Deutsche Friedhofsgesellschaft, 2021.

Der Hamburger Friedhof Ohlsdorf eröffnete 2001 den "Garten der Frauen“: eine Anlage mit historischen Grabsteinen von Frauen, die in der Hamburger Geschichte bedeutend waren; im betreffenden Areal können Frauen auch heute eine Grabstätte für sich erwerben. - Der Trend zu thematisch definierten Friedhofsarealen folgt dem Wunsch, individuellen Eigenheiten und Zugehörigkeiten der Verstorbenen über den Tod hinaus Dauer zu verleihen, indem sie in einen Kontext von Gleichgesinnten oder Gleichgestorbenen gestellt werden. Dies bedeutet eine Abkehr sowohl von den standardisierten Reihengräbern, die sich im Laufe des 2o. Jahrhunderts in den Friedhöfen etablierten, als auch vom Familiengrab, welches am Primat der biologisch-familialen Zugehörigkeit ausgerichtet ist. In diesen Kontext gehören auch neue Möglichkeiten der individualisierten Herstellung von Särgen oder Urnen, wie zum Beispiel die Bemalung des Sarges mit Lieblingsmotiven der Verstorbenen oder die Wahl eines individualisierten Urnendesigns. 3-D-Druck-Verfahren machen es zudem möglich, Lieblingsbehältnisse oder Gegenstände von Verstorbenen als Urnen zu reproduzieren wie beispielsweise eine Truhe oder auch ein Paar Boxerhandschuhe. Entsprechende Angebote finden sich derzeit insbesondere in den USA. 
Einer solchen Friedhofs-, Sarg- oder Urnengestaltung, welche die individuellen Zugehörigkeiten und Vorlieben der Verstorbenen betont, widerspricht auf den ersten Blick ein anderer deutlicher Trend der Gegenwart, nämlich der Trend zum Gemeinschaftsgrab. Bestattungen in Gemeinschaftsgräbern haben in den letzten zwei Jahrzehnten exponentiell zugenommen. In der Stadt Zürich etwa betrafen im Jahr 1990 insgesamt rund $10 \%$ aller Bestattungen Gemeinschaftsgräber, im Jahr 2000 waren es bereits $25 \%$, und heute machen Gemeinschaftsgräber bereits rund die Hälfte aller Bestattungen aus. Eine vergleichbare Zunahme verzeichnen andere Städte wie beispielsweise Berlin.

Die Motivation zum Gemeinschaftsgrab liegt auf der Hand: Die zunehmende Mobilität und häufige Veränderungen des Lebensmittelpunkts erschweren eine kontinuierliche Grabpflege durch Familienmitglieder. Außerdem will man Hinterbliebenen keine ständigen Kosten für die Grabpflege zumuten. Und das Gemeinschaftsgrab mag auch die Trostfunktion haben, dass wir im Tod nicht allein sind: Während das traditionelle Erdreihengrab in der klaren Konturierung des Rechteckes ein isoliertes und vereinzeltes Daliegen visualisiert, wird hier das Gemeinschaftliche auch im Tod betont. Daraus ergibt sich eine besondere Spannung: Einerseits mögen Hinterbliebene angesichts der weitläufigen Rasenflächen einen individuellen, deutlich sichtbaren Ort der verstorbenen Person vermissen; andererseits können sie sich darüber hinwegtrösten mit dem Gedanken, dass in unmittelbarer Nähe viele andere Verstorbene ruhen.

Erfolgt die Entscheidung für eine Bestattung im Gemeinschaftsgrab willentlich vor dem Tode, so handelt es sich um eine individuell getroffene Entscheidung, dass es am eigenen Grab keine individuelle Kenntlichkeit geben soll. ${ }^{2}$ Bisweilen unternehmen dann Hinterbliebene den Versuch, die entindividualisierten Grabstätten - sei es in FriedWäldern oder Gemeinschaftsgräbern - zu re-individualisieren: Sie bringen zu diesem Zweck Symbole wie Steckvasen, Engel oder Kreuze an, welche die Friedhofsverwaltungen gemäß ihren Reglementen, die solches untersagen, wieder entsorgen (vgl. Abb. 84).

Ein weiteres Merkmal der Zeit ist die Einrichtung von Arealen für verschiedene religiöse Zugehörigkeiten innerhalb eines Friedhofs, so dass Verstorbene unterschiedlicher Religionen auf demselben Friedhof bestattet werden können. Zumal in Westeuropa wurden in jüngerer Zeit zahlreiche Friedhöfe um muslimische und buddhistische Grabstätten erweitert, um

2 Nicole Sachmerda-Schulz: „Eine anonyme Bestattung zwischen Individualisierung und Entindividualisierung“. In: Thorsten Benkel (Hg.): Die Zukunft des Todes. Heterotopien des Lebensendes. Bielefeld 2016, S. $303 \mathrm{ff}$. 


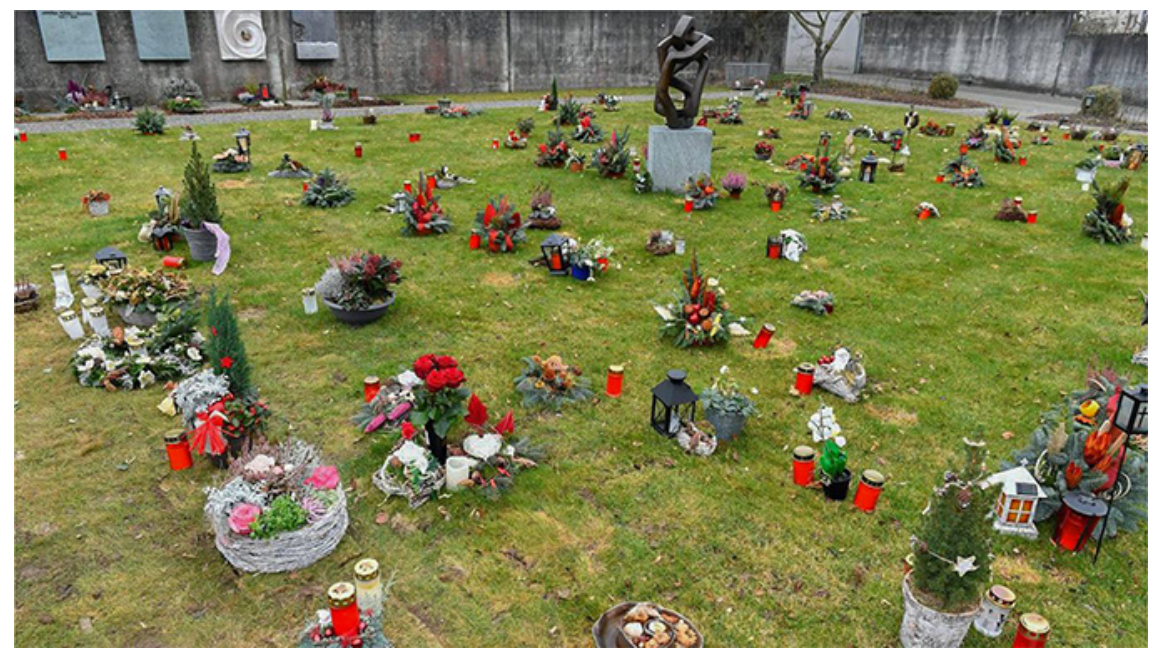

Abb. 84 Gemeinschaftsgrab, Friedhof Grenchen. Online unter: https://www. grenchnertagblatt.ch/solothurn/grenchen/blumenverbot-auf-dem-gemeinschafts grab-ein-reglement-sorgt-fuer-empoerung-134031695 (letzter Zugriff: 23.11.2021).

eine bedürfnisgerechte Gestaltung entsprechender Abschiedsorte - zum Beispiel die Errichtung von Waschräumen für rituelle Waschungen oder von Abdankungstempeln - zu ermöglichen. Mit solchen Erweiterungen soll die Diversität der städtischen Bevölkerung auch auf Friedhöfen sichtbar werden.

Insgesamt lassen sich bei den städtischen Friedhöfen eine Abkehr vom standardisierten rechteckigen Grab sowie eine Tendenz zu mehr Grünflächen mit parkähnlichem Charakter beobachten. Die Erkennbarkeit des Einzelgrabes tritt dagegen in den Hintergrund. Der heutige Friedhof strebt zur thematisch geclusterten Fläche und bietet Raum für stets neu zu definierende Communitys. Umso mehr wird er lesbar als kulturelle Signatur der Zeit, als Signatur von Lebensmodellen und Zugehörigkeiten, die über den Tod hinaus bewahrt werden. Die gesellschaftliche Auffassung des Lebens schreibt sich zunehmend auch in die Friedhöfe ein.

\section{Digitale Friedhofsseiten}

Neben der Konjunktur von Naturbestattungen und den neu strukturierten analogen Friedhöfen bietet seit nunmehr zwei Jahrzehnten auch das Internet unterschiedliche Typen von Friedhofsseiten an, die in stetiger Transformation befindlich sind. Grundsätzlich lassen sich folgende Typen unterscheiden:

- Individuelle Profilseiten auf Social-Media-Plattformen, die bei Versterben zu Gedenkseiten transformiert werden. Nach langwierigen Auseinandersetzungen mit den jeweiligen Plattformen um fehlende Rechtssicherheit sind 
die Social-Media-Gedenkseiten heute insofern reglementiert, als der Nachweis eines Totenscheins und/oder einer Erb- oder Nachlassberechtigung verlangt wird. Dennoch gestaltet sich der Prozess für Hinterbliebene teilweise nach wie vor aufwändig und nervlich strapaziös. Insbesondere die Transformation von Facebook-Profilseiten in den Memorial Mode ist oftmals außerordentlich unkomfortabel, weil sie nur von Hinterbliebenen beantragt werden kann, die selbst Mitglied bei Facebook sind.

- Virtuelle Friedhöfe oder individuelle Gedenkseiten. Solche Seiten werden in der Regel von Angehörigen eingerichtet und gepflegt, meist handelt es sich dabei um unerwartete Todesfälle (jüngere Tote, Unfalltote). Virtuelle Friedhöfe kamen Ende der 199oer-Jahre in den USA auf. Mittlerweile gibt es eine unüberschaubare Anzahl solcher Friedhöfe, die zwar noch vorhanden sind, aber nicht mehr aktualisiert und gepflegt werden, obschon die Kontaktformulare teilweise noch funktionieren und es beispielsweise zulassen, dass fiktive Todesfälle gemeldet werden.

Nach einer ersten Phase mit solchen frei zugänglichen virtuellen Friedhöfen kam ab den späten Nullerjahren ein neuer, kommerziell orientierter Friedhofstypus auf. Exemplarisch hierfür im deutschsprachigen Raum ist die Website Stayalive. Sie wurde 2010 mit einem großen medialen Paukenschlag präsentiert, der damalige Herausgeber des Nachrichtenmagazins Focus, Helmut Markwort, fungierte als Mitbegründer. Die Firma rief mit einer entsprechenden Preisliste dazu auf, sich schon zu Lebzeiten das eigene digitale Grab zu schaufeln und dieses auch zu bezahlen - in der Gewissheit, dass die Grabstätte dann nach dem Tod aufgeschaltet werde (vgl. Abb. 85).

Heute sucht man das Angebot vergeblich, die Website ist nicht mehr online. Wie bei vielen anderen ähnlichen Beispielen hat das Businessmodell "gestalte dein eigenes Grab im Internet und bezahle dafür zu Lebzeiten“ - wohl nicht funktioniert.

- Digitale Nachlassverwaltung. Hierbei handelt es sich um ein neueres Angebot, das sich derzeit ausbreitet und dessen Durchsetzungskraft noch wenig absehbar ist. Das Angebot besteht im Wesentlichen darin, OnlineDaten über Personen zusammenzutragen und dann mit den Hinterbliebenen zu entscheiden, was mit den Daten geschehen soll. Manche Anbieter fokussieren ihre Dienstleistung eher aufs Löschen von Daten, andere wiederum auf die Anlegung langfristiger Erinnerungsseiten. ${ }^{3}$ Man kann den eigenen Nachlassverwalter auch selbst vorbestimmen und bei diesem bereits zu Lebzeiten bestimmte Daten hinterlegen.

3 Z. B. semno.de oder lasthello.de (letzter Zugriff: 23.11.2021). 


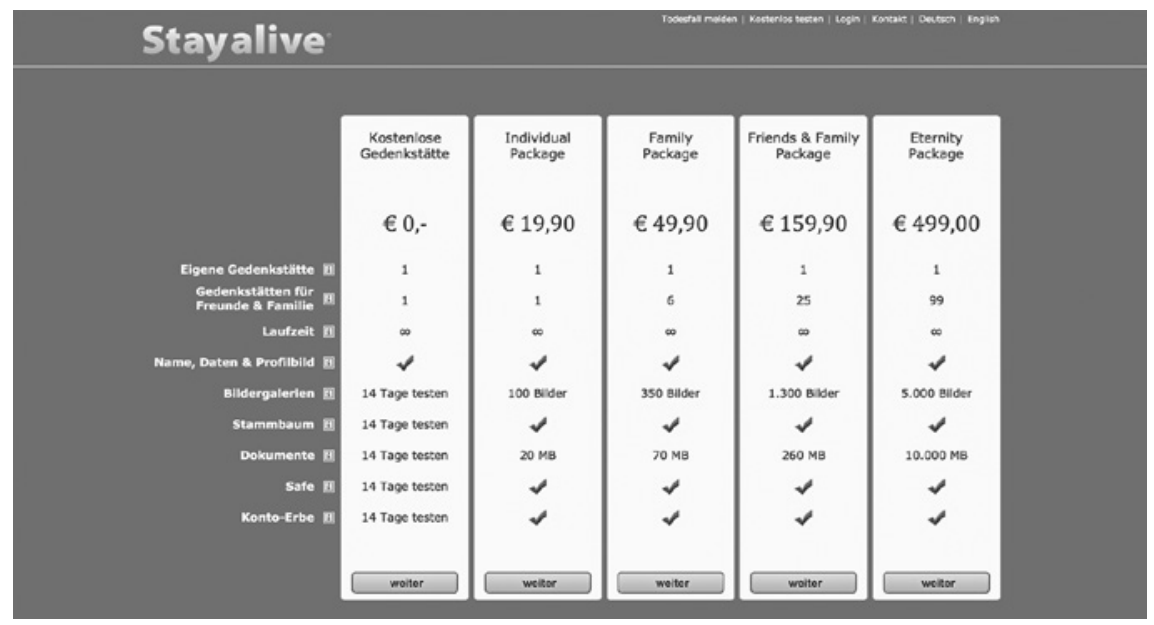

Abb. 85 Preisliste Stayalive, Screenshot vom 17.12.2012 (https://www.stayalive.com/de/ static/preise).

- Trauerportale rund um die Themen „Tod, Trauer, Erinnerung“. Solche Portale werden von karitativen Einrichtungen oder auch von Zeitungen bzw. Verlagen betrieben und bieten unterschiedliche Informationen zur Trauerbewältigung an. So suchen sie etwa den Austausch zwischen Trauernden zu fördern, oder sie offerieren Ratgeberfunktionen. Finanziert werden sie durch Anzeigen, u. a. von Bestattungsunternehmen, Florist:innen und Anbieter:innen von Gedenkobjekten. ${ }^{4}$

\section{Komplementarität}

Die verschiedenen Typen und Angebote von digitalen Gedenkseiten befinden sich, wie die Internet-Landschaft insgesamt, permanent in Umgestaltung: Hosts und Domainnamen verschwinden, neue tauchen auf, die Websprachen verändern sich. Applikationen, die gestern in aller Munde waren, sind heute nicht mehr anwendbar; gestern besuchte Webseiten lassen sich heute nicht mehr aufrufen. So wandelt sich im Netz auch die Erscheinungsweise von Gedenkkulturen permanent. Sie ist nicht dauerhaft, sondern fragil, sie kann keinen ,ewig gültigen Eintrag' garantieren, sondern zeugt vielmehr von ständiger Veränderung. ${ }^{5}$

4 Z. B. https://aspetos.com; https://trauer.sueddeutsche.de; https://trauer.nzz.ch (letzter Zugriff: 23.11.2021).

5 Michael Arnold, Martin Gibbs, Tamara Kohn, James Meese, Björn Nansen: Death and Digital Media. London 2018, S. 35f. 
Die Dynamik im Digitalen steht der analogen Friedhofskultur gegenüber, in der Gräber grundsätzlich für lange Zeit stabil und statisch angebracht sind. Damit einher geht auch die in den analogen Friedhof eingebaute Schwellenerfahrung: Das schwere gusseiserne Friedhofstor symbolisiert den Übergang in einen anderen mentalen Zustand: Wie beim Betreten einer Kirche verlangsamt man auch hier seinen Schritt, man wird bedächtig, tritt sachte auf und ermahnt Kinder zur Ruhe - der analoge Friedhof erzeugt Momente der inneren Einkehr.

Nicht so digitale Grabstätten. Sie machen keine Angebote für mentale Ruhezustände, sondern erscheinen im Gegensatz dazu permanent nervös, dauernd bewegt sich etwas: Sternchen blinken auf, brennende Kerzen segeln vorüber, liebliche Engelchen schlagen mit den Flügeln, Namenszüge verändern im Takt die Farbe - Animation ist alles. Nicht zu vergessen die oft eingespeiste süßlich-sphärische Musik. Eine kontemplative Betrachtungsweise ist nahezu ausgeschlossen. Die Vorstellung von Vergänglichkeit, von Knochen oder Asche kommt hier nicht auf, das Netz kennt keinen toten Körper. Die OnlineMediatisierung von Erinnerungskultur erfolgt niederschwellig, unverbindlich und zeitlich sowie örtlich ungebunden. Das Resultat ist eine "fluide Diskursgemeinschaft, die [...] wenig sozial normiert und reglementiert erscheint". 6

Aufgrund der zahlreichen Differenzen liegt es nahe, analoge und virtuelle Friedhöfe nicht als konkurrierende, sondern als einander ergänzende Orte zu sehen: Die innere Einkehr am realen Grab und die strenge Reglementierung analoger Friedhöfe wird komplementiert durch animierte Interaktionen und sozialen Austausch, durch rasch wandelbares Design und freie Gedenkgestaltung im Netz.

\section{Der QR-Grabstein an der Schnittstelle von analogem und digitalem Friedhof}

Ein neues Objekt, in dem sich die ko-existenzielle Komplementarität von analogem und digitalem Friedhof manifestiert, ist der so genannte QR-Grabstein. Hierzu wird an einem realen Grabstein auf einem Friedhof mittels Stein-, Metall- oder Glasapplikation ein QR-(Quick Response-)Code angebracht, welcher mit dem Mobiltelefon eingelesen werden kann. Der Code führt zu online hinterlegten Informationen über die verstorbene Person, die auf dem realen Grabstein keinen Platz haben, wie zum Beispiel Fotos oder längere Texte (vgl. Abb. 86).

6 Anke Offerhaus: „Klicken gegen das Vergessen - Die Mediatisierung von Trauer- und Erinnerungskultur am Beispiel von Online-Friedhöfen“. In: Thomas Klie, Ilona Nord: Tod und Trauer im Netz. Mediale Kommunikationen in der Bestattungskultur. Stuttgart 2016, S. 37-62: 57 . 


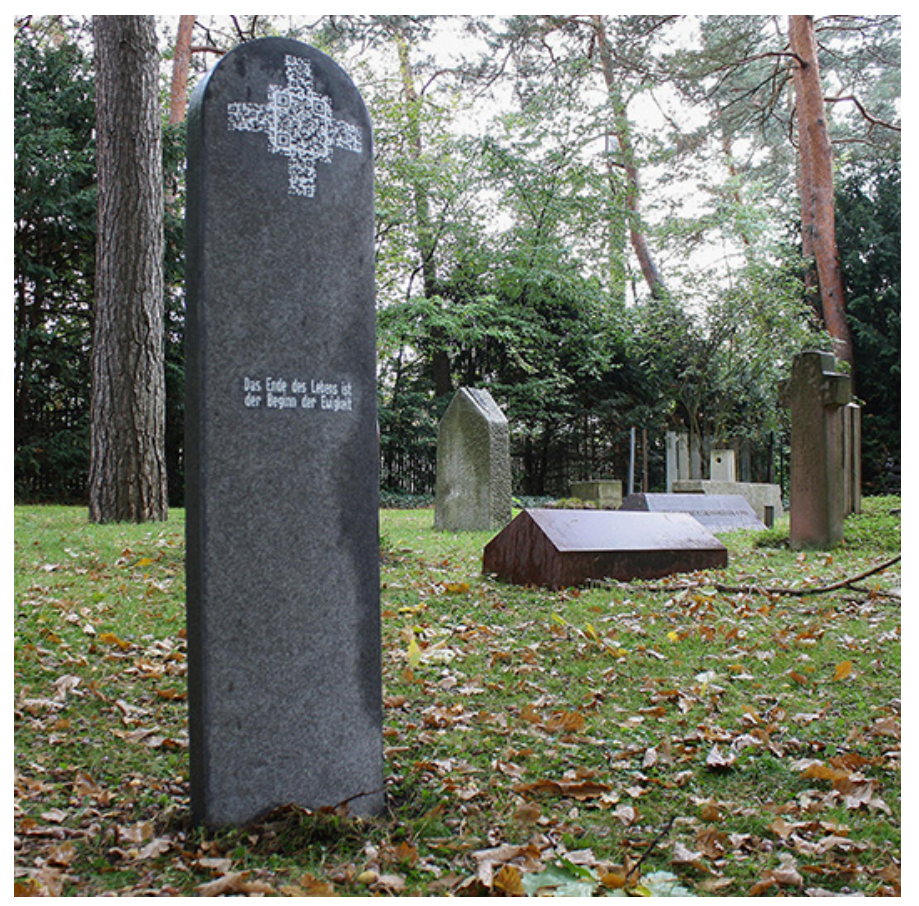

Abb. 86 QR-Grabstein, Leitfriedhof Nürnberg. Online unter: https://upload.wikimedia.org/wikipedia/commons/e/e8/ QR-Grabstein_steinart-2012.jpg (letzter Zugriff: 23.11.2021).

Der Trend kam 2008 in Japan auf. Drei Jahre später entwickelte der deutsche Steinmetz Andreas Rosenkranz das weltweit erstmalige Verfahren, den QRCode mittels Sandstrahltechnik direkt in Naturstein anzubringen, was als besonders dauerhaft gilt. Wer den Code erstellt, legt dabei die Inhalte fest und bleibt für diese verantwortlich - so zumindest handhaben es verschiedene Friedhofsverwaltungen, um für Inhalte nicht haftbar gemacht werden zu können. Denn auch hier gilt: kein digitaler Inhalt bleibt unverändert. Missbrauch - also, wenn man so will, digitale Grabschändung - ist möglich.

Ob sich der QR-Grabstein durchsetzen wird, scheint fraglich. In Deutschland löste der erste QR-Grabstein 2012 zahlreiche Medienberichte aus, aber seither scheint das Interesse wieder abgeflaut. In Zürich z. B. gibt es bis heute keine solche Grabsteine; der Verband Schweizer Bildhauer- und Steinmetzmeister VSBS teilte mit, dass zwar vereinzelte QR-Grabsteine erstellt wurden, jedoch sei ein „Trend zu QR-Grabzeichen in der Schweiz nicht zu erkennen.“7

7 E-Mail von Doris Reber, Geschäftsstelle Verband Schweizer Bildhauer- und Steinmetzmeister VSBS, an Ruedi Trachsel, Berner Fachhochschule, vom 17. Januar 2020. 


\section{Bestattungswesen und Innovation}

Zu den Aufgaben von Bestatter:innen gehören die Leichenversorgung, die Unterstützung der Hinterbliebenen bei den notwendigen Behördengängen sowie das Arrangement der Bestattung. Bestatter:innen sind somit zentrale Akteur:innen des Kulturwandels im Bestattungswesen. Aktuell zu beobachten sind neue digitale Plattformen, auf denen man die gewünschte Form der Bestattung bestellen kann, wie beispielsweise mymoria.de: Qua Internet eingegangene Bestattungsaufträge werden anschließend in Zusammenarbeit mit lokalen Bestattungsunternehmen durchgeführt.

In der Schweiz gab es in jüngster Zeit verschiedene Presseartikel über das seit 2017 aktive Online-Unternehmen bestattungsplaner.ch. Es wird vom Bestatter Johannes Ruchti betrieben, der im November 2019 in Luzern auch eine Bestattermesse organisierte. Das Unternehmen wirbt mit dem Slogan: "Wir digitalisieren die Bestattungsbranche“, doch die konventionelle Bestatterszene reagierte wenig angetan. So meinte etwa Rolf Arnold vom Luzerner Bestattungsinstitut Arnold und Sohn: „Die Gesetzmäßigkeiten des Marketings in der Bestattungsbranche kann man nicht mit anderen Unternehmen gleichsetzen." ${ }^{8}$ Eine vergleichbare Aussage findet sich in einer Studie über Death and Digital Media (2018), in welcher Bestattermessen in den USA, im UK und in Australien untersucht wurden. Darin wird ein Unternehmer zitiert, der wenig erfolgreich versuchte, mit der Bestattungsindustrie zusammenzuarbeiten: „I have worked in a variety of industries and have to say that funeral directors are the most stubborn, stuck in the mud bunch [die sturste und verstockteste Bande] I have ever met."

Tatsächlich zeichnet sich das Bestattungswesen gemäß dieser Studie gesamthaft kaum als innovationsstark aus, sondern als konservativ und risikoscheu. Dafür sind insbesondere folgende Gründe geltend zu machen ${ }^{10}$ :

- Alter und akute Trauersituation der Kundschaft. Die Kundschaft von Bestattungsunternehmen befindet sich in der Regel im Ausnahmezustand, herauskatapultiert aus dem Normalleben in einen Zustand des akuten Verlusts, in dem es eine Bestattung auszurichten gilt. Dabei steht der Anspruch auf Sicherheit und Verlässlichkeit im Vordergrund. Zudem ist diese Kundschaft in der Regel älter. Man kann sich vorstellen, dass Eltern von jungen Verstorbenen eher bereit sind, sich auf neuere Produkte einzulassen, etwa auf farbige oder bemalte Särge oder auf Urnen mit einem

\footnotetext{
8 https://www.luzernerzeitung.ch/zentralschweiz/luzern/wer-frueh-bucht-stirbt-billigerso-wirbt-ein-horwer-bestattungsinstitut-ld.111950o (letzter Zugriff: 23.11.2021).

$9 \quad$ Arnold et al. (2018): Death and Digital Media, S. 101. Übers. CC.

10 Vgl. ebd., Kap. 6: „The Funeral as a Site of Innovation“, S. 98-123.
} 
etwas extravaganteren Design. Vielleicht lassen sie auch eher zu, dass bei der Trauerfeier digitale Gadgets eingesetzt werden, die den Verstorbenen wichtig waren. Für ältere Tote hingegen, die den Regelfall darstellen, wird dies heute noch kaum beansprucht.

- Allgemeine Vergangenheitsorientierung. Tod und Bestattung bringen per se eine Rückbesinnung auf die Vergangenheit mit sich. Der Fokus auf ein vergangenes Leben steht der Innovation, die stets zukunftsorientiert ist, grundsätzlich entgegen. Im Moment des Trauerfalls ist man gemeinhin wenig ansprechbar für neuartige Produkte und mag sich in der Regel kaum lange mit entsprechenden Kaufentscheidungen befassen, denn Trauer und Kommerz vertragen sich schlecht. So zieht man sich in diesem außergewöhnlichen Moment auf Bekanntes und Standardisiertes zurück - und entscheidet sich dann etwa am liebsten für den Urnenklassiker aus gebranntem Ton.

- Familienbetriebe. Viele Bestattungsunternehmen sind Familienbetriebe, wie man es auch aus den TV-Serien Six Feet Under oder Der Bestatter kennt. Der entschleunigte Duktus überträgt sich von einer Generation zur nächsten, wohingegen Familienbetriebe in anderen Bereichen im Hinblick auf Zukunftssicherung ständig auf Innovationen setzen müssen. Im Bestattungswesen gibt es zudem vergleichsweise wenig personale Fluktuation, was sich tendenziell ebenfalls innovationshemmend auswirkt. Neigt die Bestatterszene aus den diversen Gründen grundsätzlich kaum zu Risiko- und Innovationsfreudigkeit, so ist es wenig überraschend, dass die Digitalisierung sich hier langsamer durchsetzt als in anderen Branchen. Eine Ausnahme hiervon stellt unkonventionelle Werbung im Internet dar, mit der sich heute einige Bestattungsunternehmen von der Konkurrenz, welche ihre Dienstleistungen nach wie vor mit stereotypen, von symbolischer Lichtmetaphorik geprägten Naturbildern bewirbt, abzusetzen versuchen. So wirbt etwa die digitale Plattform mymoria.de mit einem vollbepackten Auto, das die letzte Reise symbolisiert, oder einer Kreditkarte zwischen den gefalteten Händen, die für faire Bestattungskosten steht (vgl. Abb. 87-89).

Generell jedoch ist in dieser Branche die Eintrittsbarriere für neue Produkte und insbesondere für digitale Angebote hoch. Dies gilt beispielsweise auch für Live-Streaming-Bestattungen, wie sie in den USA und Australien von Angehörigen gelegentlich beansprucht werden, wenn sie eine weite Reise zur Bestattung nicht auf sich nehmen können oder wollen.

So befinden sich Bestattungspraktiken heute insgesamt in einem vielschichtigen Umbruch: Sie sind experimentell, nervös, teilweise prekär, ins Digitale überlappend. Es ist kaum vorauszusagen, wie sie sich in den kommenden Jahrzehnten weiter entwickeln werden, und es wäre überaus aufschlussreich, etwas tun zu können, was uns allen versagt ist: in hundert Jahren an einer Bestattung teilzunehmen. 


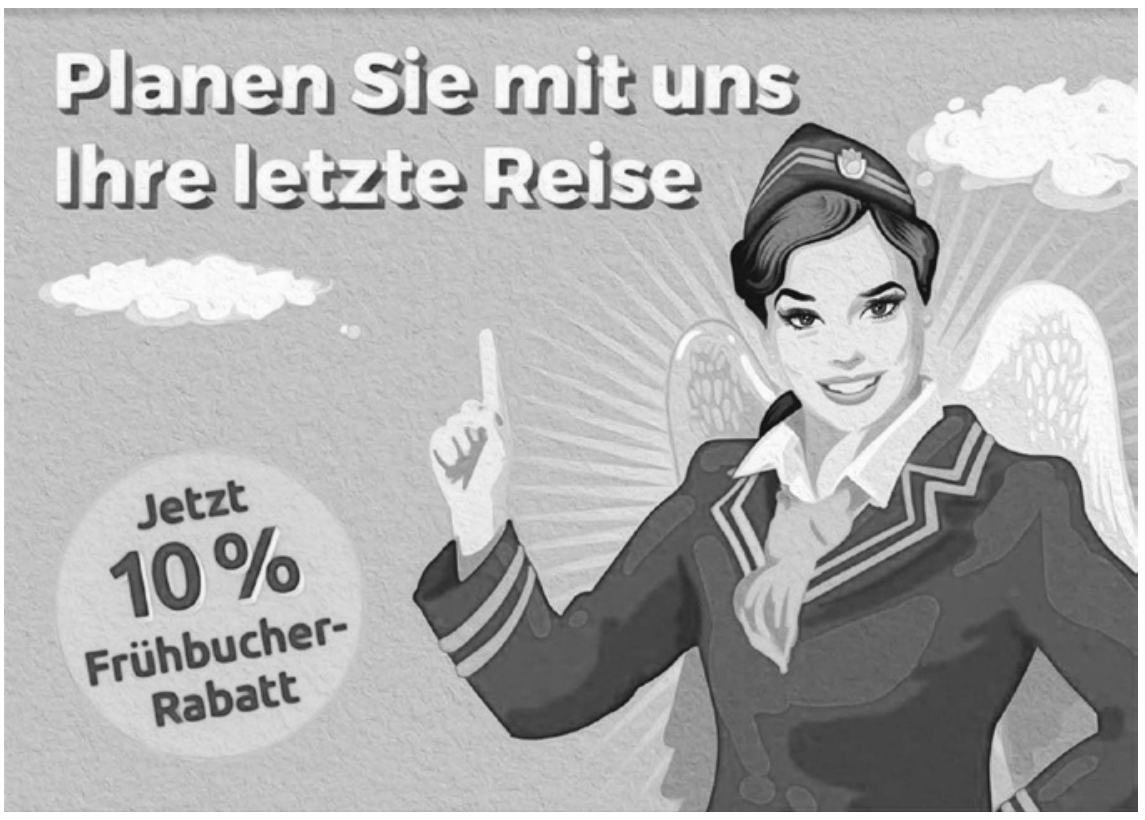

Abb. 87 Anzeigen Bestattungsunternehmen im Internet. Planen Sie mit uns Ihre letzte Reise. Online unter: https://www.2omin.ch/story/bestattungsinstitut-gib t-fruehbucherrabatt-850861192791 (letzter Zugriff: 23.11.2021).

Abb. 88

MyMemoria, Sie buchen Ihre Reise online. Online unter: https://awards. die-zeitungen.de/gewinner-anzeige-desjahres/anzeige-des-jahres-gewinner2019/\#contentSlider-1-1

(letzter Zugriff: 23.11.2021).

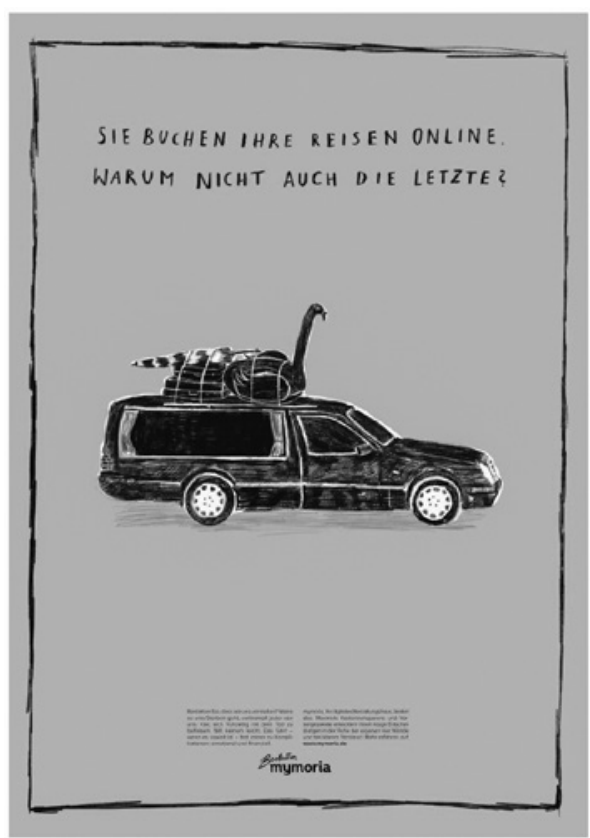




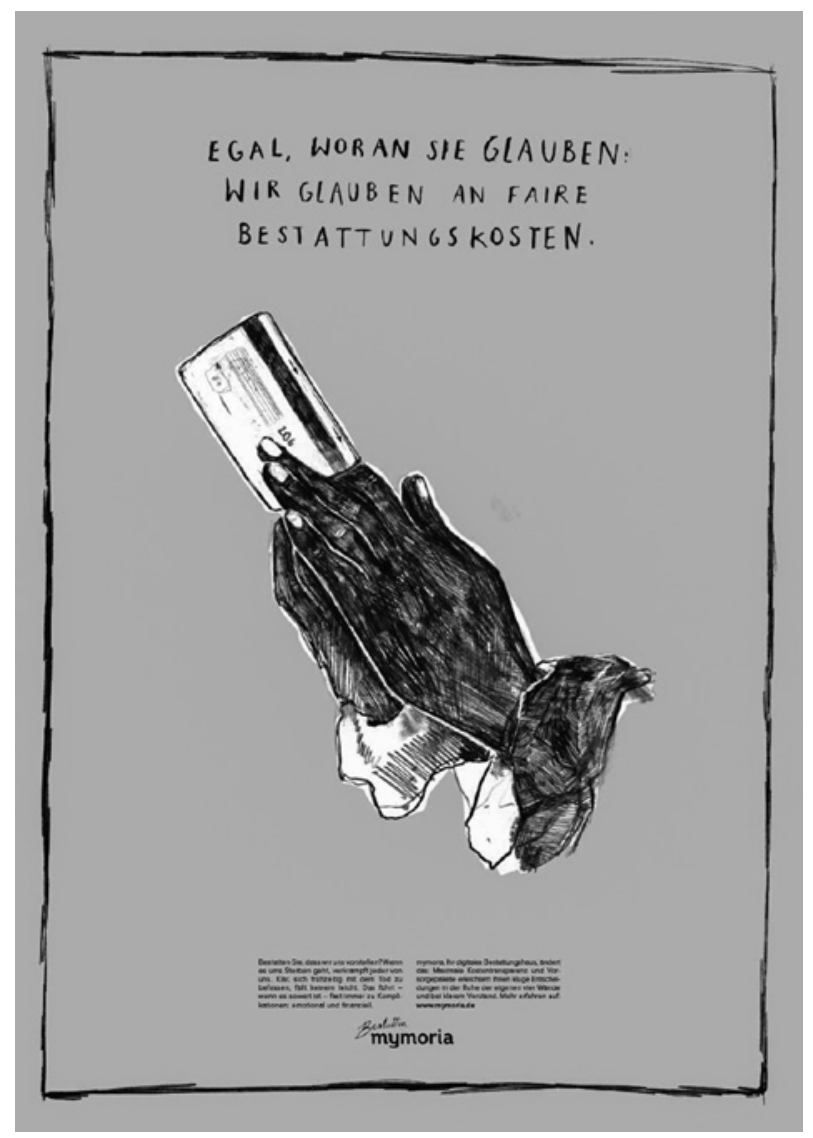

Abb. 89 MyMemoria, Egal, woran Sie glauben. Wir glauben an faire Bestattungen. Online unter: https://www. tessasima.com/mymoria-1 (letzter Zugriff: 23.11.2021). 